\title{
I risultati dei Trials randomizzati controllati sul trattamento medico della calcolosi renale sono sufficienti oggi per suggerire linee guida da usare nella pratica clinica?
}

Commento all'articolo: "Medical management to prevent recurrent nephrolithiasis in adults: a systematic review for an American College of Physicians Clinical Guideline”

\section{Renata Caudarella}

Professore Associato Malattie del Metabolismo e del Ricambio. Maria Cecilia Hospital, GVM Care and Research, Cotignola (RA)

In questa review (1) gli Autori hanno preso in considerazione un aspetto non ancora ben definito del trattamento medico della calcolosi renale che comprende sia regole comportamentali, soprattutto abitudini alimentari, sia l'uso di farmaci.

Gli Autori hanno esaminato accuratamente la bibliografia e hanno incluso solo trials randomizzati controllati (RCTs) relativi al trattamento farmacologico e/o dietetico per un totale di 28 RCTs ( 8 alimentari e 20 farmacologici). La maggior parte dei RCTs (23) includevano prevalentemente pazienti con calcolosi renale calcica, 2 si riferivano a pazienti con calcolosi di struvite e 2 includevano calcolosi di tipo diverso. La prima difficoltà nel comparare i RCTs, è lo scarso numero degli stessi unitamente alla mancanza di omogeneità degli outcomes identificati in alcuni trials come "recidive litiasiche sintomatiche, o radiologiche o composite". Quest'ultimo aspetto viene segnalato peraltro anche dagli stessi Autori. Anche il tempo del trattamento era molto variabile da 1 a 5 anni; questo periodo corrisponde al $35 \%$ di recidiva nei soggetti non trattati a distanza di 5 anni di un episodio litiasico sintomatico. Se a distanza di 5 anni senza trattamenti specifici la possibilità di recidiva è del $35 \%$ quanto può influire su questa percentuale un diverso periodo di trattamento ad esempio 1 anno di dieta e/o di terapia (diuretici tiazidici, allopurinolo, citrati alcalini) vs 5 anni? Ma altri aspetti devono essere considerati: ad esempio la tipologia delle alterazioni metaboliche rilevate (i CTRs comprendevano una identica valutazione laboratoristica?), e/o la presenza di precedenti trattamenti invasivi (ad esempio, ESWL, ureterolitotrissia, percutanea). E ancora più semplicemente, nella progettazione di un RCT chi non suggerirebbe anche ai pazienti litiasici del gruppo non trattato, di aumentare l'apporto di acqua e di mantenere una diuresi di 2 litri al giorno? Eventuali modificazioni dei comportamenti alimentari suggerite, ad esempio, dalla composizione chimica dei calcoli e/o da alterazioni metaboliche osservate (classico esempio l'ipercalciuria idiopatica) sono alla luce delle più recenti conoscenze razionalmente corrette? Sicuramente il materiale a nostra disposizione appare non più sufficiente; infatti i risultati degli studi più recenti del metabolismo minerale, delle osteopatie metaboliche, delle malattie cardiovascolari e di altre patologie che rientrano nel grande quadro della sindrome metabolica, hanno modificato il modo di affrontare il problema del trattamento/prevenzione della malattia litiasica. Ad esempio, nei pazienti con calcolosi calcica e ipercalciuria idiopatica, la dieta deve fornire un adeguato apporto di calcio (800-1000 mg/die) tenendo conto anche della concentrazione di 25-idrossicolecalciferolo che deve essere sufficiente, per consentire un adeguato assorbimento intestinale di calcio. La quota di calcio deve essere preferibilmente di natura alimentare per evitare i rischi connessi all'uso di supplementi di sali di calcio, ben descritti ad esempio da Bolland et al, nel 2010 (2) a carico dell'apparato cardiovascolare con un aumento significativo del numero di infarti del miocardio. Per quanto riguarda l'influenza dei supplementi di calcio, Jackson et al (3) hanno descritto nel Women's Health Initiative un incremento del rischio di calcolosi renale nel $17 \%$ di donne randomizzate che assumevano $1 \mathrm{~g}$ di calcio più vitamina $\mathrm{D}$. Va in questo senso anche un nostro lavoro che mostrava che i supplementi di calcio agivano nei soggetti normali analogamente al calcio alimentare; viceversa, nei pazienti calcolotici si osservava un aumento tendenziale dell' $\mathrm{AP}(\mathrm{CaOx})$ e per questo motivo consigliavamo, nel caso pazienti calcolotici dovessero assumere supplementi di calcio, un attento monitoraggio del loro $\mathrm{AP}(\mathrm{CaOx})(4)$.

Anche il ruolo delle proteine è stato rivalutato perché molti lavori che collegavano alle proteine un aumento dell'escrezione urinaria del calcio, si riferivano a diete non normoproteiche ma iperproteiche, con conseguenti effetti negativi anche sull'osso. Sebbene un elevato apporto di carne o di proteine induca un aumento del carico acido renale e dell'escrezione urinaria di calcio, recenti contributi non confermano l'asserzione che l'osso sia l'origine del surplus di calcio eliminato nelle urine. Inoltre mancano prove certe che un elevato apporto di proteine, incluse quelle di origine animale, abbiano influenza sul bilancio del calcio corporeo o contribuiscano allo sviluppo di osteoporosi e di un aumento del rischio di fratture. Sembra che l'aumento dell'assor- 
bimento di calcio, l'incremento delle concentrazioni di IGF-I, e la riduzione del PTH sierico siano in grado di compensare ogni effetto negativo del carico acido associato con un elevato apporto di proteine, sulle condizioni di "salute" dell'osso. Cao et al, concludono che l'effetto calciuretico delle proteine di origine animale, non debba essere considerato come elemento chiave tale da richiedere una precisa raccomandazione di ridurre l'apporto di proteine incluso quello che deriva dalla carne (5).

Per quanto riguarda un'altra alterazione di comune riscontro nei pazienti con calcolosi calcica, e cioè l'ipocitraturia non si può parlare di questa alterazione metabolica solo in termini di fattore di rischio per la calcolosi renale e degli effetti positivi dei citrati alcalini (soprattutto citrato di potassio, citrato di potassio e magnesio) su questa patologia. Fin dalle prime segnalazioni degli anni '90, è stata ipotizzata un'azione positiva della supplementazione dei citrati alcalini sul bilancio del calcio nelle donne dopo la menopausa. Questi concetti hanno trovato conferma in lavori molto recenti come quello di Moselkey et al (6) che hanno osservato in un gruppo di pazienti trattati con citrato di potassio, una riduzione statisticamente significativa della escrezione urinaria di calcio, un miglioramento significativo nel bilancio del calcio, una riduzione della concentrazione di PTH unitamente ad una riduzione dei markers del riassorbimento osseo (CTX). A questi risultati vanno aggiunti quelli del lavoro di Jehle et al (7) che hanno trattato un gruppo di anziani con osteopenia per 24 mesi con citrato di potassio unitamente a calcio e vitamina $\mathrm{D}$, ottenendo un aumento significativo della aBMD ossea e del vBMD trabecolare con miglioramento della microarchitettura ossea. Un'azione efficace antifratturativa sembra essere suggerita dalla riduzione del rischio di frattura valutato con l'algoritmo FRAX; quest'ultimo aspetto necessita un trial multicentrico ben strutturato con precisi outcomes.

Se gli aspetti relativi ai suggerimenti dietetici riportati in questi RCTs appaiono attualmente insufficienti, un giudizio analogo si adatta anche ai RCTs in cui sono stati utilizzati farmaci e/o citrati alcalini. Infatti gli outcomes non sono simili, non è possibile effettuare un confronto dei risultati ottenuti con farmaci diversi, e risulta strano che non siano stati riportati, ad eccezione di un trial, effetti collaterali (ad esempio, nei pazienti trattati con tiazidici non è stata mai osservata una riduzione del potassio e/o dei citrati; né si è osservato un aumento dell'uricemia o della glicemia).

In conclusione quanto sopra riportato suggerisce la necessità di impostare nuovi TRCs con outcomes primari e secondari ben definiti tenendo conto che la calcolosi renale si intreccia con numerosi aspetti del metabolismo minerale e della sindrome metabolica. È auspicabile, che nel trattamento medico di questi pazienti si debba avere una visione olistica e non limitata alla sola sintomatologia acuta, o all'imaging radiologico o ad una singola alterazione metabolica.

Dichiarazione di conflitto di interesse: L'Autore dichiara di non avere conflitto di interessi.

Contributi economici agli Autori: L'Autore dichiara di non avere ricevuto sponsorizzazioni economiche per la preparazione dell'articolo.

Indirizzo dell'Autore:

Prof.ssa Renata Caudarella

Via Aurelio Saffi 73/2

40131 Bologna

renata.caudarella@gmail.com

\section{Bibliografia}

1. Fink HA, Wilt TJ, Eidman KE, et al. Medical management to prevention recurrent nephrolithiasis in adults: a systematic review for an American College of Physicians. Clinical guideline. Ann Intern Med 2013; 158(7): 535-43.

2. Bolland MJ, Avenell A, Baron JA, et al. Effects of calcium supplements on risk of myocardial infarction and cardiovascular events; metaanalysis. BMJ 2010; 341: c3691.

3. Jackson RD, LaCroix AZ, Gass M, et al; Women's Health Initiative Investigators. Calcium plus vitamin D supplementation and the risk of fractures. N Engl J Med 2006; 354: 669-83.

4. Caudarella R, Buffa A, Rizzoli E, Sinicropi G, Vescini F. Influence of oral calcium supplements on urinary supersaturation for CaOx and $\mathrm{CaP}$ in normal and stone formers. In Eurolithiasis. $9^{\mathrm{TH}}$ European Symposium on Urolithiasis. 13-15 September 2001, Rotterdam; 129-31.

5. Cao JJ and Nielsen FH. Acid diet (high-meat protein) effects on calcium metabolism and bone health. Current Opinion in Clinical Nutrition and Metabolic Care 2010, 13: 698-702.

6. Moselkey KF, Weaver CM, Appel L, Sebastian A and Sellmeyer DE. Potassium Citrate Supplementation Results in Sustained Improvement in Calcium Balance in Older Men and Women. JBMR 2013; 28: 497-504.

7. Jehle S, Hulter HN, and Krapf R. Effect of potassium citrate on Bone Density, Microarchitecture, and Fracture Risk in Healthy Older Adults without osteoporosis: A Randomized Controlled Trial. J Clin Endocrinol Metab 2013; 98: 207-17. 